\title{
Management of respiratory disorders in a Chinese medicine teaching clinic in Australia: review of clinical records
}

\author{
Wan Najbah Nik Nabil", Wenyu Zhou, Johannah Linda Shergis, Suzi Mansu, Charlie Changli Xue \\ and Anthony Lin Zhang
}

\begin{abstract}
Background: People seek Chinese medicine (CM) treatments for a variety of respiratory disorders, e.g., asthma and upper respiratory tract infection (URTI). No previous studies have reviewed the data available in medical records from Australian clinics. This study aims to identify the characteristics of patients with respiratory disorders who visited a CM teaching clinic at RMIT University in Melbourne, Australia.

Methods: Primary data from January 2010 to December 2011 were collected from patient records in a CM teaching clinic at RMIT University. Patient data, including demographics, primary complaint, clinical history, lifestyle, CM treatment, and adverse events, were analyzed with descriptive statistics and the Chi square test using SPSS version 21.0.

Results: From 1677 clinical records we identified 261 patients with respiratory disorders. The patients made a total of 842 visits (mean: 3.2 visits/patient; range: 1-34 visits) during the study period. The mean age of the patients was $38.5 \pm 17.9$ years, and the majority were female (65.5\%). The most common respiratory disorders were URTI (27.8 \%), cough (20.8\%), hay fever or allergic rhinitis (18.6\%), sinus congestion (11.2\%), and asthma (7.6\%). Acupuncture was given at almost all visits (97.5\%) and was frequently combined with herbs (64.0\%). Fifteen adverse events were reported, but none were considered severe.
\end{abstract}

Conclusion: In the CM teaching clinic, respiratory conditions were a common presenting complaint of patients, and were safely treated with a combination of acupuncture and herbs.

\section{Background}

While the Australian government spends more than AUD 3 billion annually on respiratory disorders, $19 \%$ of Australian patients visited medical practitioners for respiratory disorders in 2007 and 2008 [1]. At least one-fifth of people with respiratory disorders, such as asthma and chronic obstructive pulmonary disease, seek complementary and alternative treatments, including acupuncture, herbal medicine, and lifestyle advice [2, 3]. However, little is known about the patient characteristics, clinical applications, and respiratory conditions treated in Chinese medicine $(\mathrm{CM})$ clinics. The respiratory disorders

\footnotetext{
*Correspondence: s3352798@student.rmit.edu.au

Traditional and Complementary Medicine Research Program, Discipline of Chinese Medicine, School of Health Sciences, RMIT University, PO Box 71, Bundoora, VIC 3083, Australia
}

included in this study were conditions affecting any part of the airways, including the nasal cavities, throat, larynx, pharynx, trachea, and lung [4].

Clinical records provide valuable data on the characteristics of patient populations in a health clinic [5] and provide insights into their therapies [6] and adverse events [7]. Knowledge of patient demographic characteristics would facilitate practitioners in including CM treatments [8]. Examination of documented adverse events can facilitate practitioners to learn about the risks of treatments [9], reduce adverse events [7], and report adverse events [9].

The RMIT University CM teaching clinic in Melbourne, Australia is open to the public. Patients are treated by senior students in their final undergraduate or postgraduate year, under the supervision of qualified CM practitioners registered with the CM Board of 
Australia. Available health services include needle acupuncture (with manual or electrical stimulation), laser acupuncture, Chinese herbal medicines (raw, granular, and manufactured), therapeutic massage, moxibustion, cupping, dietary advice, and lifestyle advice [10]. Chinese herb formulae are prepared in the clinic according to the prescription of the practitioners. At the time of the study, the patients were charged AUD 30 and 25 for initial and subsequent consultations, respectively. The consultation charges covered acupuncture treatment, therapeutic massage treatment, and cupping. However, the charges for Chinese herbal medicines varied and were dependent on the specific treatments [11].

In Australian CM clinics, the numbers of patients presenting with respiratory disorders are unknown. This study aims to identify the characteristics of patients with respiratory disorders who visited the RMIT CM teaching clinic.

\section{Methods}

\section{Study settings}

This study was a retrospective review and analysis of the clinical records of patients who received treatment for respiratory disorders at the RMIT University CM teaching clinic between January 2010 and December 2011. Patients signed informed consent forms on their first visit to the teaching clinic (Additional file 1). The signed consent form allowed students and staff at the CM clinic to use de-identified clinical records for learning and research purposes.

\section{Data source and data collection}

Data were extracted consistently by WN using a predefined data extraction form in Microsoft Excel 2007 (Microsoft, Redmond, Washington, USA). The extraction process was piloted by $\mathrm{WN}$ and independently checked by $\mathrm{WZ}$ and $\mathrm{AZ}$ to ensure data quality. Full data were collected by WN and checked by WZ and AZ to ensure accuracy and reliability.

Identifiable patient data were not extracted from the clinical records to maintain privacy and confidentiality. A unique numerical coding system was applied when extracting the free-text data to ensure consistency. The patients consented to their records being used for medical research and both the data extraction form and the study protocol were approved by the RMIT University Human Research Ethics Committee (Project number: 02/12) (Additional file 2).

Clinical records were selected when a respiratory disorder was the patient's primary complaint. Respiratory complaints included disorders of the airways including the nasal cavities, throat, larynx, pharynx, trachea, and lung [4]. The respiratory disorders were grouped according to the descriptions in the clinical records, including symptoms (e.g., runny nose) and diseases (e.g., hay fever, allergic rhinitis).

The extracted data included patient demographics (e.g., age, sex, occupation, and place of birth), primary complaint, medical history, current treatments and medications, alcohol intake, smoking history, physical activity, $\mathrm{CM}$ treatment, adverse events, and management of adverse events. A coding system was used to extract data on the CM treatment outcomes. Although we intended to assess patient responses to $\mathrm{CM}$ treatments, this was not feasible because the relevant data were not consistently recorded in the clinical records.

The original patient records did not record adverse events in a useable format for research purposes. Thus, WN extracted the events based on common terminology criteria outlined by the US National Institutes of Health [12] and evaluated the data using an intensity scale. Mild adverse events caused slight discomfort, moderate adverse events affected patients' routine activities, and severe adverse events required therapeutic management.

\section{Data analyses}

Data were expressed as mean \pm standard deviation (SD) and frequency with percentage. The data were analyzed using SPSS software (IBM SPSS statistics for windows, version 21.0; IBM Corp., Armonk, NY, USA). Missing data were left blank and excluded from the analysis. Descriptive statistics were presented for patient characteristics including demographic data, primary complaint, and clinical history. The Chi square test, indicated with a degree of freedom (df), was used to compare the patterns of respiratory disorders. Values of $P<0.05$ were considered to indicate statistically significant. Data on types of CM treatments, use of other treatment modalities, lifestyle advice, and adverse events were possible with multiple responses. For example, patients may have received a combination of CM treatments or advice or experienced more than one adverse event from a treatment.

\section{Results}

\section{General demographics}

The clinical records for a total of 1677 patients were assessed. Of these, 261 patients presented with primary respiratory disorders (Table 1 ). These 261 patients made a total of 842 visits (mean: 3.2 visits/patient; range: $1-34$ visits). Females comprised $65.5 \%$ of the patients. The mean age of the patients was 38.5 years (range: $2-100$ years). Most of the patients were born in Australia (69.6\%), with the remaining patients were born in Asia $(18.8 \%)$, Europe $(7.6 \%)$, and other continents (4.0\%). The patients were students (38.7\%), employed (42.0\%), retired (13.6\%), housewives (3.7\%), and unemployed 
Table 1 Demographics of the patients

\begin{tabular}{|c|c|}
\hline Item & n (\%) \\
\hline \multicolumn{2}{|l|}{ Number of patients } \\
\hline Total & $261(100 \%)$ \\
\hline \multicolumn{2}{|l|}{ Gender } \\
\hline Female & $171(65.5)$ \\
\hline Male & $90(34.5)$ \\
\hline \multicolumn{2}{|l|}{ Age (years) } \\
\hline $0-17$ & $19(7.3)$ \\
\hline $18-34$ & $111(42.5)$ \\
\hline $35-54$ & $71(27.2)$ \\
\hline$\geq 55$ & $60(23.0)$ \\
\hline \multicolumn{2}{|l|}{ Country of birth } \\
\hline Australia & $174(69.6)$ \\
\hline Asia & $47(18.8)$ \\
\hline Europe & $19(7.6)$ \\
\hline Other & $10(4.0)$ \\
\hline Missing & 11 \\
\hline \multicolumn{2}{|l|}{ Occupation } \\
\hline Student & $94(38.7)$ \\
\hline Employed & $102(42.0)$ \\
\hline Retired & $33(13.6)$ \\
\hline Housewife & $9(3.7)$ \\
\hline Unemployed & $5(2.1)$ \\
\hline Missing & 18 \\
\hline \multicolumn{2}{|c|}{ Distance from patients residence to the teaching clinic (km) } \\
\hline$\leq 10$ & $119(46.1)$ \\
\hline $11-35$ & $105(40.7)$ \\
\hline$\geq 36$ & $37(13.2)$ \\
\hline \multicolumn{2}{|l|}{ Chinese medicine experience } \\
\hline First time & $63(24.6)$ \\
\hline Return user & $193(75.4)$ \\
\hline Missing & 5 \\
\hline \multicolumn{2}{|l|}{ Number of visits } \\
\hline 1 & $118(45.2)$ \\
\hline $2-5$ & $100(38.3)$ \\
\hline$\geq 6$ & $43(16.5)$ \\
\hline \multicolumn{2}{|c|}{ Number of other medical conditions } \\
\hline None & $140(53.6)$ \\
\hline 1 or 2 & $43(16.5)$ \\
\hline More than 3 & $78(29.9)$ \\
\hline \multicolumn{2}{|l|}{ Use of other health care ${ }^{a}$} \\
\hline Visit to medical practitioners & $108(89.3)$ \\
\hline Supplements and herbs & $27(22.3)$ \\
\hline Chiropractic & $8(6.6)$ \\
\hline Homeopathy & $5(4.1)$ \\
\hline Osteopathy & $4(3.3)$ \\
\hline Massage & $4(3.3)$ \\
\hline Naturopathy & $4(3.3)$ \\
\hline Kinesiology & $2(1.7)$ \\
\hline Myotherapy & $1(0.8)$ \\
\hline
\end{tabular}

Table 1 continued

\begin{tabular}{ll}
\hline Item & $\mathbf{n}(\%)$ \\
\hline Meditation & $1(0.8)$ \\
Physiotherapy & $1(0.8)$ \\
Qi gong & $1(0.8)$ \\
Podiatry & $1(0.8)$ \\
Buteyko exercise & $1(0.8)$ \\
Alcohol use ( $\mathrm{n}=44)$ & \\
Nondrinker or drink rarely & $13(29.5)$ \\
Drink socially & $25(56.8)$ \\
Drink daily & $6(13.6)$ \\
Smoking ( $\mathrm{n}=35)$ & \\
Non smoker & $16(45.7)$ \\
Ex-smoker & $8(22.9)$ \\
Smoker & $11(31.4)$ \\
\hline Data analysis excludes missing values & \\
a Patients' use of other health care during January 2010 till December 2011. \\
Patients may have used more than one other health care, total \% does not add \\
up to 100\%
\end{tabular}

(2.1\%). Most patients lived within $10 \mathrm{~km}$ of the clinic (46.1\%), while $40.7 \%$ lived $11-35 \mathrm{~km}$ away and $13.2 \%$ lived further than $36 \mathrm{~km}$. Almost one-third of the patients smoked (31.4\%) and approximately one-half were social alcohol drinkers (56.8 \%). The majority of patients maintained regular exercise $(78.0 \%)$.

\section{Type and duration of respiratory disorders}

The most common visits for respiratory disorders were for upper respiratory tract infection (URTI) (47.5\%), undiagnosed cough (15.7\%), hay fever or allergic rhinitis (14.6\%), sinus congestion (13.4\%), and asthma (3.1\%) (Table 2). There was a significant difference in the number of respiratory disorders between 2010 and $2011\left(\chi^{2}\right.$ $(\mathrm{df}=14)=114.7, P<0.001)$.

The duration of the respiratory condition was reported in $78.9 \%$ of the clinical records. The reported durations were less than 2 weeks ( $36.7 \%), 2-4$ weeks (7.5\%), $5-51$ weeks (13.5\%), $1-5$ years $(16.8 \%)$, and $>5$ years (25.4\%).

Nearly one-half of the patients $(n=121 ; 46.3 \%)$ used one or more other health services during the study period. Of these, medical practitioner visits were the most commonly used health service (89.3\% of patients). Other health services included supplements and herbs $(22.3 \%)$, chiropractic $(6.6 \%)$, homeopathy $(4.1 \%)$, osteopathy (3.3\%), massage (3.3\%), and naturopathy (3.3\%).

\section{CM treatments}

Of the 261 patients with respiratory disorders, most visited the clinic once for their primary respiratory complaint (mean \pm SD: $3.23 \pm 3.95$ visits; range: $1-34$ visits). 
Table 2 Respiratory disorders

\begin{tabular}{|c|c|c|c|c|}
\hline Respiratory disorder & $\begin{array}{l}\text { Total patients, } \mathrm{n}(\%) \\
(\mathrm{N}=261)\end{array}$ & $\begin{array}{l}\text { Total visits, } \mathrm{n}(\%) \\
(\mathrm{N}=842)\end{array}$ & $\begin{array}{l}\text { Total visits } 2010, \mathrm{n}(\%) \\
(\mathrm{N}=363)\end{array}$ & $\begin{array}{l}\text { Total visits } 2011, \mathrm{n}(\%) \\
(\mathrm{N}=479)\end{array}$ \\
\hline URTI & $124(47.5)$ & $234(27.8)$ & $121(33.3)$ & $113(23.6)$ \\
\hline Cough & $41(15.7)$ & $175(20.8)$ & $74(20.4)$ & $101(21.1)$ \\
\hline Hay fever or allergic rhinitis & $38(14.6)$ & $157(18.6)$ & 65 (17.9) & $92(19.2)$ \\
\hline Sinus congestion & $35(13.4)$ & $94(11.2)$ & $47(12.9)$ & $47(9.8)$ \\
\hline Asthma & $8(3.1)$ & $64(7.6)$ & $8(2.2)$ & $56(11.7)$ \\
\hline Runny nose & $3(1.1)$ & $13(1.5)$ & $3(0.8)$ & $10(2.1)$ \\
\hline Pulmonary fibrosis & $2(0.8)$ & $22(2.6)$ & $0(0)$ & $22(4.6)$ \\
\hline Bronchitis & $2(0.8)$ & $11(1.3)$ & $5(1.4)$ & $6(1.3)$ \\
\hline Shortness of breath & $2(0.8)$ & $8(1.0)$ & $5(1.4)$ & $3(0.6)$ \\
\hline $\begin{array}{l}\text { Pulmonary alveolar } \\
\text { proteinosis }\end{array}$ & $1(0.4)$ & $32(3.8)$ & $32(8.8)$ & $0(0)$ \\
\hline Sleep apnoea & $1(0.4)$ & $12(1.4)$ & $0(0)$ & $12(2.5)$ \\
\hline Rhinitis & $1(0.4)$ & $11(1.3)$ & $1(0.3)$ & $10(2.1)$ \\
\hline Chest infection & $1(0.4)$ & $6(0.7)$ & $1(0.3)$ & $5(1.0)$ \\
\hline Pneumonia & $1(0.4)$ & $2(0.2)$ & $0(0)$ & $2(0.4)$ \\
\hline Lung cancer & $1(0.4)$ & $1(0.1)$ & $1(0.3)$ & $0(0)$ \\
\hline Total & 261 & 842 & 363 & 479 \\
\hline
\end{tabular}

Chi square test for significant difference between year 2010 and 2011: $X^{2} \quad(14)=114.7, P<0.001$

During the study period, 261 patients made a total of 842 respiratory disorder visits to the clinic

URTI upper respiratory tract infection

Comorbidities were also treated with CM as secondary complaints, and the most common secondary complaints were musculoskeletal and pain disorders (32.9\%), other respiratory disorders $(17.3 \%)$, migraine and headache $(8.8 \%)$, gastrointestinal disorders (6.5\%), and dermatological disorders $(6.2 \%)$. The majority of the patients (75.4\%) had previously experienced CM treatments.

In the 842 visits, the respiratory disorders were treated with acupuncture (97.5\%), Chinese herbs (74.2\%), cupping (7.5\%), massage (4.4\%), and moxibustion (1.1\%). The preparation types of the Chinese herbs were granules (40.7\%), manufactured (21.1\%), raw (14.1\%), and external-use wash or cream $(0.7 \%)$ (Table 3$)$.

At the majority of visits, patients were given both acupuncture and Chinese herbs (62.7 \%). The most common combinations were needle acupuncture and granule herbs (46.2\%), needle acupuncture and manufactured herbs $(22.9 \%)$, needle acupuncture and raw herbs $(17.8 \%)$, laser acupuncture and granule herbs (4.0\%), and needle acupuncture and massage (1.7 \%).

The combination of needle acupuncture and granule herbs was commonly used if the presenting conditions were pneumonia (100.0 \%), lung cancer (100.0\%), pulmonary alveolar proteinosis (71.9\%), asthma (42.2 \%), bronchitis (36.4\%), cough (30.9\%), sinus congestion (30.9\%), and URTI (27.4 \%). Meanwhile, acupuncture treatment alone was more commonly used for pulmonary fibrosis $(77.3 \%)$, sleep apnea (50.0 \%), runny nose (46.2 \%), shortness of breath (37.5\%), and hay fever or allergic rhinitis (23.6\%).

A total of 214 different acupuncture points were used. The number of points used at each treatment ranged $2-15$ (mean \pm SD $6.3 \pm 1.7$; median: 6 ). The most frequently used acupuncture points were he gu (LI4; $43.8 \%$ ), lie que (LU7; $39.5 \%$ ), zu san li (ST36; $39.5 \%$ ), feng long (ST40; $35.1 \%$ ), and ying xiang (LI20; $31.3 \%)$. The acupuncture point ying xiang (LI 20) was commonly used for sinus congestion (81.9\%), while lie que (LU7) was used for shortness of breath (62.5\%), zu san li (ST36) was used for bronchitis (72.7 \%), and ding chuan (EX-B1) was used for asthma (56.3\%).

Overall, 68 different Chinese herbal formulae were used. In the Poisons Standard 2014, the Australian Therapeutic Goods Administration outlines that certain herbs like Ephedra sinica (Ma Huang) are grouped into Schedule 4 , and can only be prescribed by authorized healthcare professionals and acquired from pharmacists upon prescription [13]. This study found that the clinic was fully compliant with the Poisons Standard 2014 by not prescribing, keeping, storing, or using herbs with toxicity. Yu Ping Feng San was the most commonly used formula (18.7 \%), followed by Xiao Qing Long Tang (7.8 \%) and Zhi Sou San (7.6 \%). In total, 152 different herbs were used. The most commonly used herbs were Radix Platycodi (Jie Geng; 51.2 \%), Radix Glycyrrhizae (Gan Cao; 41.0 \%), Rhizoma Atractylodis Macrocephalae (Bai Zhu; 
Table 3 Chinese medicine treatments

\begin{tabular}{lc}
\hline Chinese medicine treatment & $\begin{array}{c}\text { Number of visits, } \\
\mathbf{n}(\%)(\mathbf{N}=\mathbf{8 4 2})\end{array}$ \\
\hline Type of treatment $^{\mathrm{a}}$ & \\
Needle acupuncture & $779(92.5)$ \\
Granule herbs & $343(40.7)$ \\
Manufactured herbs & $178(21.1)$ \\
Raw herbs & $119(14.1)$ \\
Cupping & $63(7.5)$ \\
Ear acupressure & $51(6.1)$ \\
Laser acupuncture & $38(4.5)$ \\
Massage & $37(4.4)$ \\
Moxibustion & $9(1.1)$ \\
Externally applied herbs & $6(0.7)$ \\
Individual or combination treatment & \\
Individual treatment & $190(22.6)$ \\
Two types of treatment & $528(62.7)$ \\
Three of more types of treatment & $123(14.6)$ \\
No treatment & $1(0.1)$ \\
Advice (n = 116) & \\
Diet & \\
Exercise & $72(62.1)$ \\
Sleep, rest, relaxation or meditation & $34(29.3)$ \\
Increase water intake & $32(27.6)$ \\
Keep body warm & $25(21.6)$ \\
Other advice (such as personal hygiene, clothing, & $18(15.5)$ \\
Keep body covered from windy weather & $21(18.1)$ \\
Reduce coffee intake & $9(7.8)$ \\
Reduce alcohol intake & $8(6.9)$ \\
Reduce smoking & $7(6.0)$ \\
\hline Patints man & $3(2.6)$ \\
\hline
\end{tabular}

${ }^{a}$ Patients may have had more than one type of treatment or advice at a single visit

38.7 \%), Pericarpium Citri Reticulatae (Chen Pi; 36.7 \%), and Radix Saposhnikoviae (Fang Feng; 34.3 \%). The formulae for the most common conditions were Sang Ju Yin for URTI ( $n=18 ; 17.5 \%)$, Zhi Sou San for cough $(n=26$; $23.9 \%)$, Xin Yi San for sinus congestion ( $n=12 ; 25.0 \%)$, and Yu Ping Feng San for hay fever or allergic rhinitis $(n=34 ; 47.9 \%)$ and asthma $(n=28 ; 51.9 \%)$. A total of 23 different manufactured products were prescribed, with the most common being Bi Min Gan Wan (28.1\%), Yin Qiao San (18.5 \%), and Qian Bai Bi Yan Pian (11.2 \%).

General health and wellbeing advice was documented at a relatively small number of visits $(n=116 ; 13.8 \%)$. Lifestyle advice specific to CM comprised rest and meditation, protecting the body from windy weather, and general health approaches such as smoking cessation and exercise. Dietary advice included information on specific foods based on CM theory, and general dietary advice such as reducing coffee and alcohol, and increasing water intake (Table 3).

\section{Adverse events}

There were 15 reported adverse events from the 842 visits $(1.78 \%)$. The 15 events were experienced by 11 patients (Table 4). 12 events were mild and caused minimal discomfort and three were moderate and caused some discomfort that interfered with daily activities, but none were severe. The adverse events included diarrhea or loose stool (three events), nausea or vomiting (two events), symptom aggravation (two events), skin rash (two events), hot sensation in the body (two events), headache (one event), dizziness (one event), stomach ache (one event), and bloating (one event). Potential causality was assessed to be related to the Chinese herbs except for one event of nausea and vomiting that was potentially related to acupuncture. The practitioners responded to the adverse events by modifying the patients' treatment (e.g., changing the acupuncture points or changing the herbs or herb dosage; $53.3 \%$ ), stopping the treatment (33.3\%), or changing to another treatment (6.7\%).

\section{Discussion}

This study analyzed full clinical records from a CM teaching clinic in Australia with a specific focus on respiratory disorders. Young female students were identified as the main patient cohort visiting the teaching clinic for respiratory disorders. The most common respiratory conditions were URTI, cough, hay fever or allergic rhinitis, sinus congestion, and asthma, which are also the most common respiratory disorders encountered

\section{Table 4 Adverse events}

\begin{tabular}{lc}
\hline Adverse events & $\mathbf{n}(\%)$ \\
\hline Type of event & \\
Diarrhea or loose stools & $3(25.0)$ \\
Nausea and vomiting & $2(16.7)$ \\
Symptom aggravation & $2(16.7)$ \\
Skin rash & $2(16.7)$ \\
Hot sensation in the body & $2(16.7)$ \\
Headache & $1(8.3)$ \\
Dizziness & $1(8.3)$ \\
Stomach ache & $1(8.3)$ \\
Bloating & $1(8.3)$ \\
Practitioners' action addressing adverse events & \\
Modify treatment regime & $8(53.3)$ \\
Stop the treatment & $5(33.3)$ \\
Provided a new Chinese medicine treatment & $1(6.7)$ \\
Missing data & $1(6.7)$ \\
Total & $15(100)$ \\
\hline &
\end{tabular}


in Australian general practice [14]. Most visits were for acute episodes or symptoms that were present for less than 2 weeks. However, at least two-fifths of the patients had their condition for more than 1 year and attended the clinic for an acute flare-up. A large number of the visits involved treatment by both acupuncture and herbal medicine, suggesting that a combined CM intervention was common for the treatment of respiratory disorders.

Previous studies [15-18] reported that most patients were employed and middle-aged, while students and young people (age: 18-34 years) represented the largest proportion of patients in the present study. The teaching clinic examined is located on a university campus, and many of the patients were young students. The patients were more likely to be female, consistent with previous reports for studies on CM clinics [15-19].

The adverse event profile revealed a small number of mild and moderate adverse events, and was similar to the findings in an Australian survey on the safety of CM treatments [20]. None of the adverse events required specific intervention. However, in response to nearly half of the events, the CM treatment was modified.

This study had several limitations. The clinical records were developed for clinical purposes and their use for research and retrospective data collection was at times inconsistent and incomplete. Although the authors attempted to ensure accurate collection of information, misinterpretations or mistakes may have occurred. We could not assess the clear outcomes of the CM treatments. It was difficult to evaluate the treatment outcomes of patients owing to inconsistent documentation on their progress and the fact that some patients did not return to the clinic. In addition, the information relating to demographics, including age and employment, might change. Therefore, the results may not be generalizable or represent other clinical practices that are off-campus. The patients often used CM treatments in conjunction with other healthcare services. The clinical records did not reveal any interactions with other healthcare treatments, and the CM treatments appeared to be safe to use in this group of patients.

Future prospective studies on clinical records should be developed to meet the needs of both clinicians and researchers. These efforts could include the use of standardized clinical terminology, information on outcome-related data (e.g., validated quality of life or disease-specific questionnaires), and comprehensive documentation of adverse events.

The findings from this study may guide documentation of medical records (such as collecting data on treatment outcomes) [21], provide data for the planning of clinical research projects [22], and inform curriculum design for the CM learning program [22]. Moreover, understanding the profiles and behaviors of patients will promote the use of the clinic by potential patients and improve the treatment quality.

\section{Conclusion}

In the $\mathrm{CM}$ teaching clinic, respiratory conditions were a common presenting complaint of patients, and were safely treated with a combination of acupuncture and herbs.

\section{Additional files}

Additional file 1: Patient consent form.

Additional file 2: RMIT University Human Research Ethics Committee study approval.

Abbreviations

CM: Chinese medicine; URTI: upper respiratory tract infections.

\section{Authors' contributions}

$A Z, W N$ and CCX designed the study. WN, AZ and WZ performed the data extraction and statistical analysis. WN wrote the manuscript. JS, SM and CX revised the manuscript. All authors read and approved the final manuscript.

\section{Acknowledgements}

We thank the Malaysian Ministry of Health for providing a scholarship to one of the authors, WN. There was no other funding support for this project. We thank Dr James Baglin for his statistical advice. We also thank the clinic reception staff who helped with data collection.

\section{Competing interests}

The authors declare that they have no competing interests.

Received: 10 July 2014 Accepted: 26 October 2015

Published online: 02 November 2015

\section{References}

1. Australian Institute of Health and Welfare. Asthma, chronic obstructive pulmonary disease and other respiratory diseases in Australia. Canberra. 2010.

2. Slader CA, Reddel HK, Jenkins CR, Armour CL, Bosnic-Anticevich SZ. Complementary and alternative medicine use in asthma: Who is using what? Respirology. 2006;11:373-87.

3. George J, loannides-Demos LL, Santamaria NM, Kong DCM, Stewart K. Use of complementary and alternative medicines by patients with chronic obstructive pulmonary disease. Med J Aust. 2004;248-251. http:// www.mja.com.au.public/issues/181_205_060904/geo010146_fm.html.

4. Dorland's illustrated medical dictionary [Internet]. [Credo Reference].

5. Cooper F. A chart review from an oriental medicine college, comparing patients who came for only one visit and those who came for at least five visits. Med Acupunct. 2011;23:107-10.

6. Su K, Zhu F, Guo L, Zhu Y, Li W, Xiong X. Retrospective study on Professor Zhongying Zhou's experience in Traditional Chinese Medicine treatment on diabetic nephropathy. J Tradit Chin Med. 2013;33:262-7.

7. Lal LS, Gerber DL, Lau J, Dana W. Retrospective evaluation of weekly paclitaxel hypersensitivity reactions reported utilizing an electronic medical record system at a tertiary cancer center. Support Care Cancer. 2009;17:1311-5.

8. Armstrong AR, Thiébaut SP, Brown LJ, Nepal B. Australian adults use complementary and alternative medicine in the treatment of chronic illness: a national study. Aust N Z J Public Health. 2011;35:384-90. 
9. Wu T, Shang H, Bian Z, Zhang J, Li T, Li Y, Zhang B. Recommendations for reporting adverse drug reactions and adverse events of Traditional Chinese Medicine. J Evid Based Med. 2010;3:11-7.

10. Chinese Medicine Teaching Clinic. http://www.rmit.edu.au/ browse; $\mid \mathrm{D}=\mathrm{y} 3 \mathrm{bfmn} 043 \mathrm{aigz}$.

11. Chinese Medicine Teaching Clinic. http://www.rmit.edu.au/chinese-med/ teaching-clinic.

12. National Cancer Institute (US). Common Terminology Criteria for Adverse Events (CTCAE); 2009.

13. Therapeutic Good Administration (TGA). Poisons standard 2014. In: Book poisons standard. Australian Government Department of Health; 2014

14. Britt H, Miller G, Charles J, Henderson J, Bayram C, Valenti L, Harrison C, Pan Y, O'Halloran J, Zhang C, Fahridin S. General practice activity in Australia 2010-2011. Sydney: Sydney University Press; 2011.

15. Maiers M, McKenzie E, Evans R, McKenzie M. Patient outcomes at a Traditional Chinese Medicine teaching clinic: a prospective data collection project. J Altern Complement Med. 2008;14:1083-8.

16. Xing $M$, Long AF. A retrospective survey of patients at the University of Salford Acupuncture Clinic. Complement Ther Clin Pract. 2006;12:64-71.

17. Kim KH, Kim YR, Noh SH, Kang KW, Kim JK, Yang GY, Lee BR. Use of acupuncture for pain management in an academic Korean medicine hospital: a retrospective review of electronic medical records. Acupunct Med. 2013;31:228-34.
18. Vincent A, Kruk KM, Cha SS, Bauer BA, Martin DP. Utilisation of acupuncture at an academic medical centre. Acupunct Med. 2010;28:189-90.

19. Bullock ML, Pheley AM, KiresukTJ, Lenz SK, Culliton PD. Characteristics and complaints of patients seeking therapy at a hospital-based alternative medicine clinic. J Altern Complement Med. 1997;3:31-7.

20. Bensoussan A, Myers SP, Carlton AL. Risks associated with the practice of traditional Chinese medicine: an Australian study. Arch Fam Med. 2000;9:1071-8.

21. Maiers M, McKenzie E, Evans R, McKenzie M. The development of a prospective data collection process in a traditional Chinese medicine teaching clinic. J Altern Complement Med. 2009;15:305-20 (cited 8 Mar 2013).

22. Meier $P C$, Rogers $C$. Reporting traditional Chinese medicine morbidity - a University of Technology, Sydney, Project with an emphasis on developing standards for testing and reporting data. J Altern Complement Med. 2006;12:529-34 (cited 6 Jun 2013)

\section{Submit your next manuscript to BioMed Central and take full advantage of:}

- Convenient online submission

- Thorough peer review

- No space constraints or color figure charges

- Immediate publication on acceptance

- Inclusion in PubMed, CAS, Scopus and Google Scholar

- Research which is freely available for redistribution

Submit your manuscript at

www.biomedcentral.com/submit

C Biomed Central 